\title{
Accurate non-Born-Oppenheimer calculations of the complete pure vibrational spectrum of ditritium using all-particle explicitly correlated Gaussian functions
}

\author{
Sergiy Bubin, ${ }^{1}$ Monika Stanke, ${ }^{2}$ and Ludwik Adamowicz ${ }^{3}$ \\ ${ }^{1}$ Department of Chemistry, University of Rochester, Rochester, New York 14627, USA \\ ${ }^{2}$ Institute of Physics, Faculty of Physics, Astronomy and Informatics, Nicolaus Copernicus University, \\ Grudziądzka 5, 87-100 Toruń, Poland \\ ${ }^{3}$ Department of Chemistry and Biochemistry, University of Arizona, Tucson, Arizona 85721, USA and \\ Department of Physics, University of Arizona, Tucson, Arizona 85721, USA
}

(Received 7 March 2014; accepted 30 March 2014; published online 16 April 2014)

\begin{abstract}
Very accurate variational calculations of the complete pure vibrational spectrum of the ditritium $\left(\mathrm{T}_{2}\right)$ molecule are performed within the framework where the Born-Oppenheimer approximation is not assumed. After separating out the center-of-mass motion from the total laboratory-frame Hamiltonian, $\mathrm{T}_{2}$ becomes a three-particle problem. States corresponding to the zero total angular momentum, which are pure vibrational states, are spherically symmetric in this framework. The wave functions of these states are expanded in terms of all-particle, one-center, spherically symmetric explicitly correlated Gaussian functions multiplied by even non-negative powers of the internuclear distance. In the calculations the total energies, the dissociation energies, and expectation values of some operators dependent on interparticle distances are determined. ( 2014 AIP Publishing LLC. [http://dx.doi.org/10.1063/1.4870935]
\end{abstract}

\section{INTRODUCTION}

Treating the nuclei and electrons on equal footing and not assuming the clamped nucleus approximation makes the problem of calculating stationary, bound states of a molecular system much more complicated than the problem of determining bound states of electrons in the field of stationary nuclei, as it is done in calculations where the Born-Oppenheimer (BO) approximation is assumed. In non-BO calculations one deals with describing three types of correlation effects, i.e., the electron-electron, nucleus-nucleus, and nucleus-electron correlations. This places more demands on the basis functions which are used to expand the wave function representing a stationary state of the system. Also, for a non-BO calculation to be relevant it has to describe the system very accurately and, thus, the basis functions have to be capable of providing very accurate representation of the considered state. This can only happen if the basis functions used in the non-BO calculations explicitly depend on the distances between all pairs of particles forming the system.

The system we consider in the calculations performed in the present work is the $T_{2}$ hydrogen isotopologue. The basis functions for expanding the non-BO wave functions corresponding to the pure vibrational states of $T_{2}$ are multiparticle explicitly correlated Gaussian functions (ECG). It has been shown that the exponential dependence on the interparticle distances of these functions facilitates very effective description of the inter-electron correlation effects. Also, as the Gaussian functions have maxima at zero inter-particle distances, they are adequate for describing the nucleus-electron correlation. However, the strong nuclear-nuclear correlation is more difficult to describe with Gaussians only dependent on the inter-particle distances in the exponent because due to their repulsion and much heavier masses the wave function practically vanishes when two nuclei approach each other. To describe this behavior we multiply the all-particle ECGs by powers of the internuclear distances (by the internuclear distance in the case of a diatomic molecule). ${ }^{1-3}$ The basis functions of this kind have been shown to very effectively represent zero-angular-momentum bound states of small diatomics. ${ }^{45}$ They are also effective in describing the oscillations of the wave function which arise when the system becomes vibrationally excited.

The $\mathrm{T}_{2}$ molecule is a good model for testing non-BO calculations because its pure vibrational spectrum includes as many as 27 bound states. The main contribution to the wave function of the highest excited 27th state has 26 nodes. As in the non-BO calculations the coupling of the vibrational and electronic motions is explicitly included, the wave function may also include some minor contributions with nodes in terms of both the electron-nucleus and electron-electron distances. This effect is called the nonadiabatic state mixing in the approach based on the BO approximation. As in our nonBO approach no restrictions (other than the symmetry restrictions) are imposed on the wave function, the state mixing is automatically permitted to occur in the calculation. With this full accounting for the adiabatic and non-adiabatic effects is directly obtained in the calculation and one does not need to resort to the perturbation theory to account for these effects as it is done in the approach based on the $\mathrm{BO}$ approximation.

Recently we calculated the two lowest pure vibrational states of $T_{2}$ using a basis set of 10000 ECGs for each state ${ }^{6}$ using the non-BO approach. In this work the non-BO calculations are performed for all 27 bound pure vibrational states of this system. The sizes of the basis sets used range from 11000 for the lowest $v=0$ state to 16000 for the 25th state. In the 
non-BO calculations we are using a non-relativistic Hamiltonian obtained by rigorously separating out the motion of the center of mass from the laboratory-frame Hamiltonian. The "internal" Hamiltonian obtained this way is rotationally invariant and its eigenfunctions transform according to the irreducible representations of the group of 3D rotations $(\mathrm{SO}(3))$. In particular, the ground state or any rotationless $N=0$ state of a system with positive (natural) parity is represented by a spherically symmetric wave function, which can be expanded in terms of spherically symmetric ECGs. All zero-angularmomentum bound states (i.e., the pure vibrational states) of $\mathrm{T}_{2}$ are such states.

The presentation of the results begins with a brief description of the method used in the calculations (a more complete description of the method can be found in our recent reviews ${ }^{1,2}$ ). The results obtained in the calculations are presented and discussed in Sec. III.

\section{THE METHOD USED IN THE CALCULATIONS}

In this work we consider all existing 27 bound rotationless states of the $\mathrm{T}_{2}$ molecule. The standard Rayleigh-Ritz variational method is employed to minimize the internal energy and to optimize the wave function for each state. Each state is calculated independently. The rigorous separation of the center-of-mass kinetic energy from the laboratory-frame Hamiltonian results in the internal nonrelativistic Hamiltonian, $\hat{H}_{\text {nonrel }}$, which for $\mathrm{T}_{2}$ has the following form:

$$
\begin{aligned}
\hat{H}_{\text {nonrel }}= & -\frac{1}{2}\left(\sum_{i=1}^{3} \frac{1}{\mu_{i}} \nabla_{\mathbf{r}_{i}}^{2}+\sum_{i=1}^{3} \sum_{j \neq i}^{3} \frac{1}{m_{0}} \nabla_{\mathbf{r}_{i}} \cdot \nabla_{\mathbf{r}_{j}}\right) \\
& +\sum_{i=1}^{3} \frac{q_{0} q_{i}}{r_{i}}+\sum_{i<j}^{3} \frac{q_{i} q_{j}}{r_{i j}} .
\end{aligned}
$$

In (1), $q_{0}=q_{1}=1$ are the charges of the nuclei and $q_{2}=q_{3}$ $=-1$ are the electron charges, $\mathbf{r}_{i}, i=1,2,3$, are the position vectors of the second nucleus and the two electrons with respect to the first nucleus (placed in the center of the internal coordinate system; we call this nucleus the "reference particle"), $r_{i}$ are their lengths, $r_{i j}=\left|\mathbf{r}_{j}-\mathbf{r}_{i}\right|, m_{0}=m_{1}$ $=5496.92158 m_{e}$ are the triton masses, $m_{2}=m_{3}=m_{e}=1$ are the electron masses, ${ }^{7}$ and $\mu_{i}=m_{0} m_{i} /\left(m_{0}+m_{i}\right)$ is the reduced mass of particle $i$. One can describe Hamiltonian (1) as representing three "pseudoparticles" with charges equal to the charges of the original particles, but with masses being the reduce masses, moving in the central potential of the charge of the reference particle. The motions of the three pseudoparticles are coupled through the Coulomb interactions and through the mass-polarization terms, $-\frac{1}{2} \sum_{i=1}^{3} \sum_{j \neq i}^{3} \frac{1}{m_{0}} \nabla_{\mathbf{r}_{i}}$. $\nabla_{\mathbf{r}_{j}}$.

As mentioned, spherically symmetric ECGs that include even non-negative powers of the internuclear distance, $r_{1}$, as preexponential multipliers are used in expanding the spatial parts of the non-BO wave functions of the rotationless states of $\mathrm{T}_{2} \cdot{ }^{3}$ These ECGs have the following form:

$$
\phi_{k}=r_{1}^{m_{k}} \exp \left[-\mathbf{r}^{\prime}\left(A_{k} \otimes I_{3}\right) \mathbf{r}\right]
$$

where $\mathbf{r}=\left\{\mathbf{r}_{1}^{\prime}, \mathbf{r}_{2}^{\prime}, \mathbf{r}_{3}^{\prime}\right\}^{\prime}$ and ${ }^{\prime}$ denotes the vector (matrix) transposition. $A_{k}$ in (2) is a symmetric $2 \times 2$ matrix. The $m_{k}$ power ranges from 0 to 250 in the present calculations.

The appropriate symmetry with respect to electron and nucleus permutations is imposed on basis functions (2). As the present calculations concern states which have singlet multiplicities for the electrons and for the nuclei, each basis function is made symmetric with respect to the permutations of both the electrons and nuclei. Since the transformation between the laboratory and the internal coordinates is linear, the symmetrization operators, which originally are defined with respect to the laboratory coordinates, can be expressed in terms of the internal coordinates and directly applied to functions (2). ${ }^{3}$

As mentioned, 10000 ECGs were used for each state in the previous calculations concerning the lowest two pure vibrational states of $\mathrm{T}_{2} \cdot{ }^{6}$ In this work these two basis sets are increased to 11000 for the $v=0$ state and to 12000 for the $v=1$ state. The increase is done by adding new functions to the set. The functions are added one by one in subsets of 100 and their non-linear parameters are optimized by the variational energy minimization. Each time the parameters of the optimized function are changed, the generalized eigenvalue problem is solved to determine the linear expansion coefficients and to assure that the total energy is an upper bound to the exact nonrelativistic energy of the state considered in the calculation.

The initial form of the newly added function is generated by randomly perturbing the non-linear parameters of some most contributing functions already included in the basis set and choosing the function which lowers the energy the most. After the addition of each 100-ECG subset to the basis set the whole set is reoptimized. In this reoptimization the ECGs are again optimized one by one. Such an approach allows for better control over linear dependencies between ECGs, which may arise in the optimization, because each reoptimized function can be checked for linear dependencies with all other functions in the set and, if any are found, the function is returned to its original form it had before the reoptimization.

The ECGs used in the calculations have to be square integrable. This automatically happens if $A_{k}$ is represented in a Cholesky-factored form, $A_{k} \equiv L_{k} L_{k}^{\prime}$, with $L_{k}$ being a lower triangular real matrix. The non-zero matrix elements of $L_{k}$ can vary in the $(-\infty,+\infty)$ range. The elements of the $L_{k}$ matrices are the nonlinear parameters which are optimized for each basis functions. Additionally, the $m_{k}$ power of $r_{1}$ of the function is also optimized. This is done only once after the function is first included in the basis set. The nonlinearparameter optimization is the most time consuming step of the calculation. To expedite the optimization process for the $L_{k}$ matrix elements the analytical gradient of the energy functional determined with respect to these elements is used in the optimization.

The above-described procedure is also applied to generate the basis sets for the remaining $v=2, \ldots, 26$ states. Again, for each state the basis set is generated in a separate calculation. At the start of the calculation, 10000 ECGs generated for the state located just below the considered state are included in the basis set and their $L_{k}$ parameters are thoroughly 


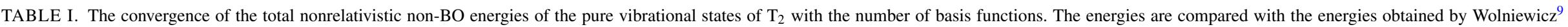

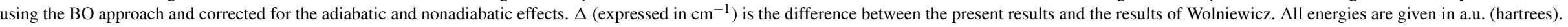

\begin{tabular}{|c|c|c|c|c|c|c|c|c|c|c|c|c|c|c|}
\hline No. ECGs & $v$ & Energy & $v$ & Energy & $v$ & Energy & $v$ & Energy & $v$ & Energy & $v$ & Energy & $v$ & Energy \\
\hline 10000 & \multirow[t]{5}{*}{0} & -1.16853567568 & \multirow[t]{5}{*}{1} & -1.15730657768 & \multirow[t]{5}{*}{2} & -1.14644188340 & \multirow[t]{5}{*}{4} & -1.13593600147 & \multirow[t]{5}{*}{5} & -1.12578421545 & \multirow[t]{5}{*}{6} & -1.11598271359 & \multirow[t]{5}{*}{7} & -1.10652862771 \\
\hline 11000 & & -1.16853567571 & & -1.15730657779 & & -1.14644188369 & & -1.13593600190 & & -1.12578421601 & & -1.11598271431 & & -1.10652862877 \\
\hline 12000 & & & & -1.15730657787 & & -1.14644188389 & & -1.13593600223 & & -1.12578421646 & & -1.11598271488 & & -1.10652862962 \\
\hline Wolniewicz & & -1.16853563156 & & -1.15730653220 & & -1.14644183770 & & -1.13593595607 & & -1.12578093010 & & -1.11598266996 & & -1.10652858698 \\
\hline$\Delta$ & & 0.0097 & & 0.0100 & & 0.0101 & & 0.0101 & & 0.0101 & & 0.0097 & & 0.0094 \\
\hline 10000 & \multirow[t]{7}{*}{7} & -1.09742008804 & \multirow[t]{7}{*}{8} & -1.08865628067 & \multirow[t]{7}{*}{9} & -1.08023753782 & \multirow[t]{7}{*}{10} & -1.07216542733 & \multirow[t]{7}{*}{11} & -1.06444288954 & \multirow[t]{7}{*}{12} & -1.05707435658 & \multirow[t]{7}{*}{13} & -1.05006590209 \\
\hline 11000 & & -1.09742008913 & & -1.08865628210 & & -1.08023753959 & & -1.07216543003 & & -1.06444289229 & & -1.05707436373 & & -1.05006593107 \\
\hline 12000 & & -1.09742008995 & & -1.08865628324 & & -1.08023754097 & & -1.07216543222 & & -1.06444289448 & & -1.05707436863 & & -1.05006595461 \\
\hline 13000 & & & & & & & & & & & & -1.05707437218 & & -1.05006597274 \\
\hline 14000 & & & & & & & & & & & & & & -1.05006598106 \\
\hline Wolniewicz & & -1.09742004958 & & -1.08865624639 & & -1.08023750796 & & -1.07216540570 & & -1.06444287336 & & -1.05707435545 & & -1.05006598678 \\
\hline$\Delta$ & & 0.0089 & & 0.0081 & & 0.0072 & & 0.0058 & & 0.0046 & & 0.0029 & & -0.0013 \\
\hline 10000 & \multirow[t]{8}{*}{14} & -1.04342576041 & \multirow[t]{8}{*}{15} & -1.03716360567 & \multirow[t]{8}{*}{16} & -1.03129260999 & \multirow[t]{8}{*}{17} & -1.02582941210 & \multirow[t]{8}{*}{18} & -1.02079128762 & \multirow[t]{8}{*}{19} & -1.01620195062 & \multirow[t]{8}{*}{20} & -1.01208921302 \\
\hline 11000 & & -1.04342577656 & & -1.03716392302 & & -1.03129324961 & & -1.02582945074 & & -1.02079132671 & & -1.01620200261 & & -1.01208926908 \\
\hline 12000 & & -1.04342579098 & & -1.03716399948 & & -1.03129334997 & & -1.02582948359 & & -1.02079135915 & & -1.01620204529 & & -1.01208931680 \\
\hline 13000 & & -1.04342580050 & & -1.03716402590 & & -1.03129338091 & & -1.02582950327 & & -1.02079138485 & & -1.01620207324 & & -1.01208935012 \\
\hline 14000 & & -1.04342580745 & & -1.03716403810 & & -1.03129339853 & & -1.02582951638 & & -1.02079140468 & & -1.01620209307 & & -1.01208936807 \\
\hline 15000 & & & & & & & & -1.02582952361 & & -1.02079141219 & & -1.01620210384 & & -1.01208937999 \\
\hline Wolniewicz & & -1.04342581144 & & -1.03716404919 & & -1.03129342128 & & -1.02582954993 & & -1.02079145115 & & -1.01620214708 & & -1.01208943335 \\
\hline$\Delta$ & & -0.0009 & & -0.0024 & & -0.0050 & & -0.0058 & & -0.0086 & & -0.0095 & & -0.0117 \\
\hline 10000 & \multirow[t]{9}{*}{21} & -1.00848662094 & \multirow[t]{9}{*}{22} & -1.00543457625 & \multirow[t]{9}{*}{23} & -1.00298225194 & \multirow[t]{9}{*}{24} & -1.00118853168 & \multirow[t]{9}{*}{25} & -0.10001224145 & \multirow[t]{9}{*}{26} & -0.99981745016 & & \\
\hline 11000 & & -1.00848668027 & & -1.00543466020 & & -1.00298229989 & & -1.00118858633 & & -1.00012243870 & & -0.99981823470 & & \\
\hline 12000 & & -1.00848672809 & & -1.00543470805 & & -1.00298235321 & & -1.00118862478 & & -1.00012245500 & & -0.99981836088 & & \\
\hline 13000 & & -1.00848676200 & & -1.00543476202 & & -1.00298238461 & & -1.00118865452 & & -1.00012246764 & & -0.99981839262 & & \\
\hline 14000 & & -1.00848678312 & & -1.00543478803 & & -1.00298240505 & & -1.00118867193 & & -1.00012247692 & & -0.99981839996 & & \\
\hline 15000 & & -1.00848679166 & & -1.00543480753 & & -1.00298241544 & & -1.00118868393 & & -1.00012248323 & & -0.99981840217 & & \\
\hline 16000 & & & & & & & & -1.00118869249 & & & & & & \\
\hline Wolniewicz & & -1.00848684329 & & -1.00543486966 & & -1.00298247658 & & -1.00118873123 & & -1.00012251629 & & & & \\
\hline$\Delta$ & & -0.0113 & & -0.0136 & & -0.0134 & & -0.0085 & & -0.0073 & & & & \\
\hline
\end{tabular}


TABLE II. Dissociation energy for the pure vibrational states of $T_{2}$ in $\mathrm{cm}^{-1}$. The energies are obtained by subtracting the doubled total energy of two tritium atoms of -0.99981811308 hartree from the total energy of the particular state obtained with the largest ECG basis set generated for that state. All values are in $\mathrm{cm}^{-1}$.

\begin{tabular}{rccccr}
\hline \hline$v$ & $\begin{array}{c}\text { No. } \\
\text { ECGs }\end{array}$ & $\begin{array}{c}\text { Dissociation } \\
\text { energy }\end{array}$ & $v$ & $\begin{array}{c}\text { No. } \\
\text { ECGs }\end{array}$ & $\begin{array}{c}\text { Dissociation } \\
\text { energy }\end{array}$ \\
\hline 0 & 11000 & $37029.2249(0)$ & 14 & 14000 & $9570.7826(20)$ \\
1 & 12000 & $34564.7228(0)$ & 15 & 14000 & $8196.4831(20)$ \\
2 & 12000 & $32180.1980(0)$ & 16 & 14000 & $6908.0267(40)$ \\
3 & 12000 & $29874.4235(0)$ & 17 & 15000 & $5708.8447(50)$ \\
4 & 12000 & $27646.3641(1)$ & 18 & 15000 & $4603.1071(50)$ \\
5 & 12000 & $25495.1832(2)$ & 19 & 15000 & $3595.8703(50)$ \\
6 & 12000 & $23420.2513(2)$ & 20 & 15000 & $2693.2318(50)$ \\
7 & 12000 & $21421.1579(2)$ & 21 & 15000 & $1902.5550(50)$ \\
8 & 12000 & $19497.7246(4)$ & 22 & 15000 & $1232.7219(50)$ \\
9 & 12000 & $17650.0243(5)$ & 23 & 15000 & $694.4841(50)$ \\
10 & 12000 & $15878.4012(5)$ & 24 & 16000 & $300.8074(50)$ \\
11 & 12000 & $14183.5001(6)$ & 25 & 15000 & $66.8015(30)$ \\
12 & 13000 & $12566.2956(6)$ & 26 & 15000 & $0.0634(20)$ \\
13 & 14000 & $11028.1323(6)$ & & & \\
\hline \hline
\end{tabular}

reoptimized ( $m_{k}$ powers are not reoptimized) by cycling over all functions in the set multiple times and performing the optimization of only one function at a time. After that the basis set is grown in the same way as it was done for the $v=0$ and $v=1$ states. The growing process stops when the energy of the state does not change by more than about $0.5 \times 10^{-8}$ hartree. As the number of vibrational nodes in terms of the $r_{1}$ coordinate increases with the vibrational excitation, more ECGs are needed for the higher states than for the lower ones. The largest number of basis functions equal to 16000 is generated for the $v=24$ state. Reusing the first 10000 ECGs from the $v=k-1$ state to initiate the calculation for the $v$ $=k$ state is justified by the observation made in the analysis of the $\mathrm{H}_{2}$ basis. This analysis showed that, while for the first few pure vibrational states the $m_{k}$ powers in the preexponential ECG multipliers are mostly smaller numbers in the $0-250$ range, the powers for higher states are approximately evenly distributed in the whole range of the allowed powers. That approach was also employed in generating the initial basis sets for the top states in the $\mathrm{D}_{2}$ calculations. ${ }^{8}$

After the basis sets and the corresponding non-BO wave functions have been generated for all 27 states, which has involved several months of continuous calculations, some expectation values involving inter-particle distances are generated. The results of the calculations are shown and discussed in Sec. III.

\section{THE RESULTS}

In Table I we present the total nonrelativistic energies of all 27 pure vibrational states of $\mathrm{T}_{2}$ obtained in the

TABLE III. Some expectation values calculated for the pure vibrational states of the $\mathrm{T}_{2}$ molecule with the non-BO nonrelativistic wave functions expanded in terms of explicitly correlated Gaussians. The basis set of the largest size for each state is used. $\left\langle r_{t}-t\right\rangle,\left\langle r_{t}-e\right\rangle$, and $\left\langle r_{e}-e\right\rangle$ denote the triton-triton, triton-electron, and electron-electron distances, respectively. All values are in a.u.

\begin{tabular}{|c|c|c|c|c|c|c|c|c|c|c|c|}
\hline$v$ & $\left\langle 1 / r_{t-t}\right\rangle$ & $\left\langle 1 / r_{t-e}\right\rangle$ & $\left\langle 1 / r_{e}-e\right\rangle$ & $\left\langle r_{t-t}\right\rangle$ & $\left\langle r_{t-e}\right\rangle$ & $\left\langle r_{e}-e\right\rangle$ & $\left\langle r_{t-t}^{2}\right\rangle$ & $\left\langle r_{t-e}^{2}\right\rangle$ & $\left\langle r_{e-e}^{2}\right\rangle$ & $\left\langle\delta\left(\mathbf{r}_{t-e}\right)\right\rangle$ & $\left\langle\delta\left(\mathbf{r}_{e-e}\right)\right\rangle$ \\
\hline 0 & 0.705667 & 0.906353 & 0.582673 & 1.428359 & 1.563908 & 2.187730 & 2.056241 & 3.099443 & 5.732488 & 0.227455 & 0.016401 \\
\hline 1 & 0.689942 & 0.894605 & 0.573867 & 1.483304 & 1.592899 & 2.224135 & 2.248689 & 3.222635 & 5.928941 & 0.223405 & 0.015776 \\
\hline 2 & 0.674477 & 0.883101 & 0.565042 & 1.539472 & 1.622453 & 2.261744 & 2.452095 & 3.350946 & 6.134926 & 0.219507 & 0.015167 \\
\hline 3 & 0.659238 & 0.871822 & 0.556179 & 1.596991 & 1.652631 & 2.300708 & 2.667360 & 3.484837 & 6.351567 & 0.215763 & 0.014571 \\
\hline 4 & 0.644191 & 0.860754 & 0.547255 & 1.656013 & 1.683506 & 2.341202 & 2.895535 & 3.624849 & 6.580180 & 0.212159 & 0.013990 \\
\hline 5 & 0.629299 & 0.849877 & 0.538245 & 1.716713 & 1.715159 & 2.383427 & 3.137854 & 3.771622 & 6.822316 & 0.208696 & 0.013419 \\
\hline 6 & 0.614522 & 0.839175 & 0.529122 & 1.779301 & 1.747692 & 2.427623 & 3.395779 & 3.925918 & 7.079817 & 0.205357 & 0.012862 \\
\hline 7 & 0.599819 & 0.828628 & 0.519853 & 1.844024 & 1.781221 & 2.474070 & 3.671055 & 4.088649 & 7.354878 & 0.202153 & 0.012306 \\
\hline 8 & 0.585144 & 0.818215 & 0.510403 & 1.911176 & 1.815889 & 2.523102 & 3.965788 & 4.260916 & 7.650138 & 0.199058 & 0.011760 \\
\hline 9 & 0.570446 & 0.807913 & 0.500732 & 1.981116 & 1.851869 & 2.575119 & 4.282537 & 4.444064 & 7.968792 & 0.196084 & 0.011222 \\
\hline 10 & 0.555668 & 0.797697 & 0.490791 & 2.054278 & 1.889369 & 2.630602 & 4.624450 & 4.639747 & 8.314745 & 0.193220 & 0.010685 \\
\hline 11 & 0.540744 & 0.787539 & 0.480526 & 2.131194 & 1.928648 & 2.690136 & 4.995443 & 4.850023 & 8.692817 & 0.190459 & 0.010153 \\
\hline 12 & 0.525599 & 0.777405 & 0.469872 & 2.212528 & 1.970028 & 2.754441 & 5.400447 & 5.077486 & 9.109018 & 0.187803 & 0.009608 \\
\hline 13 & 0.510147 & 0.767258 & 0.458753 & 2.299113 & 2.013912 & 2.824413 & 5.845769 & 5.325447 & 9.570953 & 0.185224 & 0.009076 \\
\hline 14 & 0.494282 & 0.757052 & 0.447076 & 2.392011 & 2.060817 & 2.901179 & 6.339592 & 5.598200 & 10.088365 & 0.182759 & 0.008530 \\
\hline 15 & 0.477881 & 0.746734 & 0.434728 & 2.492594 & 2.111411 & 2.986182 & 6.892729 & 5.901402 & 10.673959 & 0.180381 & 0.007974 \\
\hline 16 & 0.460789 & 0.736237 & 0.421573 & 2.602683 & 2.166577 & 3.081306 & 7.519821 & 6.242694 & 11.344677 & 0.178084 & 0.007407 \\
\hline 17 & 0.442816 & 0.725478 & 0.407435 & 2.724728 & 2.227508 & 3.189061 & 8.241138 & 6.632617 & 12.123612 & 0.175881 & 0.006823 \\
\hline 18 & 0.423719 & 0.714348 & 0.392092 & 2.862129 & 2.295856 & 3.312884 & 9.085658 & 7.086180 & 13.043235 & 0.173743 & 0.006220 \\
\hline 19 & 0.403180 & 0.702709 & 0.375252 & 3.019767 & 2.373993 & 3.457648 & 10.096381 & 7.625554 & 14.150907 & 0.171677 & 0.005592 \\
\hline 20 & 0.380770 & 0.690367 & 0.356519 & 3.204950 & 2.465471 & 3.630567 & 11.340274 & 8.285102 & 15.519115 & 0.169678 & 0.004932 \\
\hline 21 & 0.355886 & 0.677047 & 0.335330 & 3.429274 & 2.575920 & 3.842961 & 12.928414 & 9.121523 & 17.266028 & 0.167751 & 0.004233 \\
\hline 22 & 0.327622 & 0.662332 & 0.310838 & 3.712672 & 2.715027 & 4.114108 & 15.062068 & 10.237025 & 19.602219 & 0.165859 & 0.003486 \\
\hline 23 & 0.294507 & 0.645528 & 0.281640 & 4.093685 & 2.901534 & 4.481026 & 18.156344 & 11.841322 & 22.955263 & 0.164022 & 0.002681 \\
\hline 24 & 0.253725 & 0.625283 & 0.245028 & 4.663328 & 3.179793 & 5.030826 & 23.276636 & 14.470149 & 28.407609 & 0.162220 & 0.001803 \\
\hline 25 & 0.197954 & 0.597997 & 0.193792 & 5.747907 & 3.709466 & 6.076568 & 34.773143 & 20.302486 & 40.330774 & 0.160444 & 0.000846 \\
\hline 26 & 0.062680 & 0.531232 & 0.062610 & 20.660465 & 11.111502 & 20.784901 & 556.355184 & 281.176733 & 562.347767 & 0.159069 & 0.000013 \\
\hline
\end{tabular}


calculations. The convergence of the energy with the number of ECGs in the basis set is shown for each state. As one can see, the convergence is noticeably better for the lower states than for the upper states despite using for larger basis sets for the latter states. The number of ECGs in the basis set varies from 11000 for the ground state to 16000 for the $v=24$ state. The present results are compared in Table I with the results of Wolniewicz ${ }^{9}$ obtained using the conventional approach employing a potential energy curve, which includes adiabatic corrections. The Wolniewicz's results are also corrected for the nonadiabatic effects. The comparison shown that, in general, the two sets of results agree with each other. However, as one notices, our energies are slightly lower by about $0.01 \mathrm{~cm}^{-1}$ for the few lowest states than the Wolniewicz's energies, then the difference decreases to eventually become negative and equal to about $-0.01 \mathrm{~cm}^{-1}$ for the top states.

In Table II, $\mathrm{T}_{2}$ dissociation energies corresponding to all bound 27 pure vibrational states are shown. For each energy value we provide an estimate of the error, which is due to the basis-set incompleteness. This incompleteness rises with the excitation level. As one can see the present non-BO calculations have been converged to a very high accuracy at the nonrelativistic level. As the non-BO approach directly and explicitly includes accounting for the finite-nuclear-mass effects in the calculations, these effects (both adiabatic and nonadiabatic) are included to very high accuracy in the energy and in the wave function. They are also accounted for in the expectation value calculations, as those values are determined using the nonrelativistic non-BO wave functions.

The calculated expectation values include the following average quantities: the inverse of the triton-triton distance, $\left\langle 1 / r_{t-t}\right\rangle$, the inverse of the triton-electron distance, $\left\langle 1 / r_{t-e}\right\rangle$, the inverse of the electron-electron distance, $\left\langle 1 / r_{e-e}\right\rangle$, the triton-triton distance, $\left\langle r_{t-t}\right\rangle$, the triton-electron distance, $\left\langle r_{t-e}\right\rangle$, the electron-electron distance, $\left\langle r_{e-e}\right\rangle$, the square of the triton-triton distance, $\left\langle r_{t-t}^{2}\right\rangle$, the square of the tritonelectron distance, $\left\langle r_{t-e}^{2}\right\rangle$, the square of the electron-electron distance, $\left\langle r_{e-e}^{2}\right\rangle$, the contact triton-electron density, $\left\langle\delta\left(\mathbf{r}_{t-e}\right)\right\rangle$, and the contact electron-electron density, $\left\langle\delta\left(\mathbf{r}_{e-e}\right)\right\rangle$. The results are shown in Table III. The expectation values show the expected trends. The average $t-t$ distance increases with the vibrational excitation. For the highest $v=26$ state, which is predicted to be marginally bound by only about $0.06 \mathrm{~cm}^{-1}$, the average $t-t$ distance is over 20 a.u. This is three times larger than for the next lower state. Same is true for the deuteron-electron and electron-electron average distances.
These results indicate that, as this state is very close to the dissociation threshold, it may involve a higher level of coupling of the motions of the electrons and the nuclei. This coupling is automatically included in our non-BO calculations.

\section{SUMMARY}

In this work we present very accurate non-BO calculations of the whole pure vibrational spectrum of the $T_{2}$ molecule performed with all-particle explicitly correlated Gaussian functions. Depending on the state the basis set size varies from 11000 to 16000 . The exponential parameters of the Gaussians are extensively optimized using the standard variational method aided with the analytically calculated energy gradient determined with respect to these parameters. The high accuracy of the calculations is achieved mainly due to the use of the gradient which significantly expedites the optimization process. As the expected accuracy of the results is very high, they may provide useful benchmark values for conventional calculations performed using the conventional BO approach based on the potential energy curve.

\section{ACKNOWLEDGMENTS}

We are grateful to the University of Arizona Research Computing Services for providing computer resources for this work. We thank Professor Wolniewicz for sending to us his unpublished $\mathrm{T}_{2}$ results. This work has been supported in part by a grant from the National Science Center (NCN) of Poland awarded to Monika Stanke.

${ }^{1}$ M. Cafiero, S. Bubin, and L. Adamowicz, Phys. Chem. Chem. Phys. 5, 1491 (2003).

${ }^{2}$ S. Bubin, M. Cafiero, and L. Adamowicz, Adv. Chem. Phys. 131, 377 (2005).

${ }^{3}$ D. B. Kinghorn and L. Adamowicz, J. Chem. Phys. 110, 7166 (1999).

${ }^{4}$ S. Bubin, M. Stanke, and L. Adamowicz, J. Chem. Phys. 131, 044128 (2009).

${ }^{5}$ M. Stanke, S. Bubin, and L. Adamowicz, Phys. Rev. A 79, 060501(R) (2009).

${ }^{6}$ S. Bubin, M. Stanke, M. Molski, and L. Adamowicz, Chem. Phys. Lett. 494, 21 (2010).

${ }^{7}$ See CODATA 2002 recommended values and NIST Physical.

${ }^{8}$ S. Bubin, M. Stanke, and L. Adamowicz, J. Chem. Phys. 135, 074110 (2011).

${ }^{9} \mathrm{~L}$. Wolniewicz, the adiabatic potential energy curve for $T_{2}$ was provided to us in a private communication; the non-adiabatic corrections for $T_{2}$ are taken from www.fizyka.umk.pl/ luwo/data/xnon_cor.95 (1995); L. Wolniewicz, J. Chem. Phys. 99, 1851 (1993); 103, 1792 (1995); W. Kolos and L. Wolniewicz, ibid. 49, 404 (1968). 